\title{
Global Training of Document Processing Systems using Graph Transformer Networks.
}

\author{
Léon Bottou, Yoshua Bengio, Yann Le Cun \\ Speech and Image Processing Services Research Lab \\ AT\&T Labs, 101 Crawfords Corner Road \\ Holmdel, NJ 07733
}

\begin{abstract}
We propose a new machine learning paradigm called Graph Transformer Networks that extends the applicability of gradient-based learning algorithms to systems composed of modules that take graphs as inputs and produce graphs as output. Training is performed by computing gradients of a global objective function with respect to all the parameters in the system using a kind of back-propagation procedure.

A complete check reading system based on these concepts is described. The system uses convolutional neural network character recognizers, combined with global training techniques to provides record accuracy on business and personal checks. It is presently deployed commercially and reads million of checks per month.
\end{abstract}

\section{Introduction}

The most common technique for building document processing systems is to partition the task into manageable subtasks, such as field detection, word segmentation, or character recognition, and to build a separate module for each one. Typically, each module is trained, or manually optimized, outside of its context. After the complete system is assembled, a subset of the parameters of the modules is manually adjusted to maximize the overall performance. This last step is extremely tedious, time-consuming, and often suboptimal.

For example, a character recognition module can be trained to recognize well-formed individual characters. However, the role of the recognizer in the context of the entire system is usually quite different. Very often, character recognizers are expected, not only to recognize wellformed individual characters, but also to reject badly segmented characters and other non-characters. They are also expected to produce reliable scores that can be used by a post-processor, such as a statistical language model. For example, if the task is to read the amount on bank checks, the overall objective function that must be minimized is the percentage of checks that must be rejected in order to attain a given error rate on the accepted checks. Merely training the character recognizer module to minimize its classification error on individual characters will not minimize the global objective function. Ideally, we would like to find a good minimum of the global objective function with respect to all the parameters in the system. In most practical cases, this optimization problem appears intractable. However, if the objective function $E$ measuring the performance measure can be made differentiable with respect to the system's tunable parameters $W$, we can find its minimum $\min _{W} E(W)$ using gradient-based techniques such as stochastic gradient descent or conjugate gradient.

A simple technique to ensure that the global objective function $E(W)$ is differentiable is to build the overall system as a feed-forward network of differentiable modules. The function implemented by each module must be continuous and differentiable almost everywhere with respect the internal parameters of the module (e.g. the weights of a Neural Net character recognizer in the case of a character recognition module), and with respect to the module's inputs. If this is the case, a simple generalization of the wellknown back-propagation procedure, widely used for multilayer neural net training, can be used to efficiently compute the gradients of the objective function with respect to all the parameters in the system [3]. For example, let us consider a system built as a cascade of modules, each of which implements a function $X_{n}=F_{n}\left(W_{n}, X_{n-1}\right)$, where $X_{n}$ is a vector representing the output of the module, $W_{n}$ is the vector of tunable parameters in the module (a subset of $W$ ), and $X_{n-1}$ is the module's input vector (as well as the previous module's output vector). If we assume that the partial derivative of $E$ with respect to $X_{n}$ is known, the partial derivatives of $E$ with respect to $W_{n}$ and $X_{n-1}$ can be computed using

$$
\begin{aligned}
\partial E / \partial W_{n} & =\partial F / \partial W\left(W_{n}, X_{n-1}\right) \partial E / \partial X_{n} \\
\partial E / \partial X_{n-1} & =\partial F / \partial X\left(W_{n}, X_{n-1}\right) \partial E / \partial X_{n}
\end{aligned}
$$

where $\partial F / \partial W\left(W_{n}, X_{n-1}\right)$ is the Jacobian of $F$ with respect to $W$ evaluated at the point $\left(W_{n}, X_{n-1}\right)$, and $\partial F / \partial X\left(W_{n}, X_{n-1}\right)$ is the Jacobian of $F$ with respect to $X$. Using the above equation, we can compute the complete gradient of $E(W)$ working our way backwards from the output to the input as in the traditional back-propagation procedure.

\section{Gradient Based Learning in Large Heterogeneous Systems}

Traditional multilayer neural networks can be viewed as cascades of trainable modules (the layers) that communicate their states and gradients in the form of fixed-size vec- 

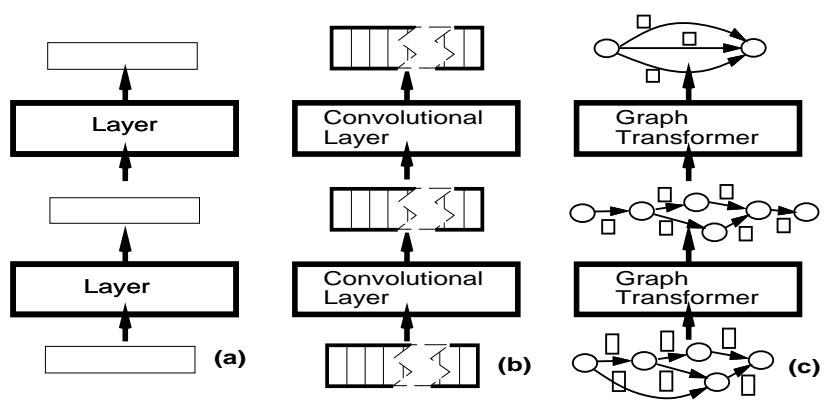

Fig ure 1. (a) Traditional Neural Networks communicate fixed-size vectors between layer. (b) Convolutional and Recurrent NN can handle variable-length sequences of vectors. (c) Multilayer Graph Transformer Networks are composed of trainable modules that operate on and produce graphs whose arcs carry numerical information.

tors. The limited flexibility of fixed-size vectors for data representation is a serious deficiency for many applications, notably for tasks that deal with variable length inputs (e.g speech and handwriting recognition), or for tasks that require encoding relationships between objects or features whose number and nature can vary (invariant perception, scene analysis, reasoning).

Convolutional network architectures such as Time Delay Neural Networks (TDNN), Space Displacement Neural Networks (SDNN) [7], or recurrent network, have been proposed to deal with variable-length sequences of vectors. They have been applied with great success to optical character recognition, on-line handwriting recognition, spoken word recognition, and time-series prediction.

However, these architectures still lack flexibility for tasks in which the state must encode more than simple vector sequences. A notable example is when the state is used to encodes probability distributions over sequences of vectors (stochastic grammars). In such cases, the data is best represented using a directed graph in which each arc contains a vector. Each path in the graph represents a different sequence of vectors. Distributions over sequences can be represented by interpreting parts of the data associated with each arc as a penalty, score, or likelihood. Distributions over sequences are particularly handy for modelling linguistic knowledge in speech or handwriting recognition systems: each sequence, i.e. each path in the graph, represents an alternative interpretation of the input. Successive processing modules progressively refine the interpretation. For example, a speech recognition system starts with a single sequence of acoustic vectors, transforms it into a lattice of phonemes (distribution over phoneme sequences), then into a lattice of words (distribution over word sequences), then into a single sequence of words representing the best interpretation.

One of the main points of the paper is to show that the gradient-based training procedure described in the previous section is not limited to networks simple modules that communicate through fixed-size vectors, but can be generalized to networks of modules called graph transformers that communicate their states and gradients in the form of graphs whose arcs carry numerical information (scalars or vectors). A Graph transformer takes one or more graphs as input and constructs a graph on its output (see figure 1). A back-propagation phase takes gradients with respect to the numerical information in the output graph, and computes gradients with respect to the numerical information attached to the input graphs, and with respect to the transformer's internal parameters. Gradient-based learning can be performed as long as differentiable functions are used to produce the numerical data in the output graph from the numerical data in the input graph, and from the functions parameters.

The second goal of the paper is to show that the functions implemented by many of the modules used in typical document processing systems (and other image recognition systems), though commonly thought to be combinatorial in nature, are indeed differentiable with respect to their internal parameters as well as with respect to their inputs, and are therefore usable as part of a globally trainable system.

Lastly, we demonstrate the use of globally-trained graphtransformer networks on a check reading task. The check reading system we describe here is commercially deployed, reading several million handwritten and machine printed checks per month with record accuracy.

\section{Graph Transformer Networks}

To help make the of graph transformer network more concrete, we will describe an oversimplified example of a trainable system built from three graph transformers in the context of handwriting recognition. The task of the system is to find the best segmentation of a handwritten word into characters (see figure 2). A word image is first cut into "pieces of ink" using heuristic image processing techniques (such as connected components, vertical projections,...). Each piece of ink may be a whole character or a piece of character. The bottom of figure 2 shows an example of a three-character word cut into four pieces of ink. A so-called segmentation graph $G_{\text {seg }}$ is built to represent all the possible groupings of pieces of ink into characters. Each arc is associated with one piece of ink or with a combination of successive pieces (called a segment), such that each path in the graph goes once and only once through each piece of ink. To each path corresponds a different grouping of the pieces of ink into characters. A first graph transformer $T_{r e c}$, which we will call the recognition transformer, creates its output graph $G_{r e c}$ by replicating the segmentation graph, replacing each segment by a positive number. If the segment is a good-looking character, this number is small (close to 0), if it is an incomplete or bad-looking character, this number is large. These numbers can be seen as negative log-likelihoods, distances, or simply penalty factors. They are generated from the segment images through a character scorer function $R_{w}$, parameterized by a vector $w$ (e.g. a neural network with weight vector $w$ ). The cumulated penalty over a path in $G_{r e c}$ is the badness (or penalty) of the corresponding segmentation. A second transformer $T_{v i t}$, called a Viterbi transformer, takes this graph as input and produces a trivial graph $G_{v i t}$ whose only path is the lowest-penalty path in the segmentation graph. A third transformer $T_{\text {cost }}$ takes $G_{v i t}$ and outputs a single number: the cumulated penalty $C$ of $G_{v i t}$. These three transformers are very special cases. $T_{r e c}$ changes the content of the arcs but not the structure of the graph. $T_{v i t}$ changes the graph 
0.8

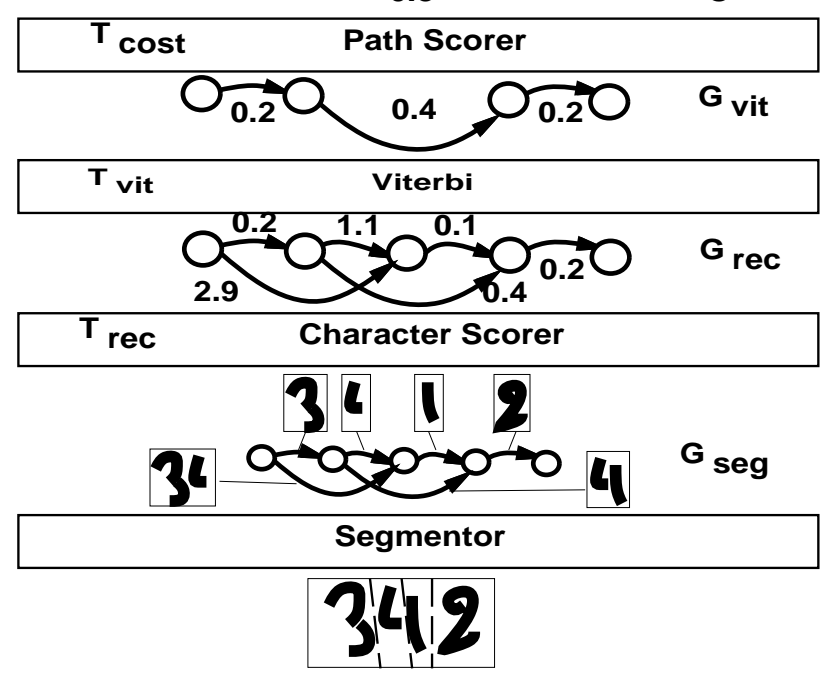

Fig ure 2. A simple example of GTN that finds the best segmentation of a handwritten word.

structure, but it simply duplicates a subset of the arcs on its output without changing their content. $T_{\text {cost }}$ outputs a single number. More complex transformer will be described in the main part of the paper.

\subsection{Backpropagation in Graph Transformer Networks}

Backpropagating gradients through the above system is very simple. We assume that the system is part of larger system whose training minimizes an objective function $E$. For each variable $x$ used the forward pass (arc penalties, character scorer parameters....), the backpropagation phase will compute a corresponding partial derivative $\partial E / \partial x$. We assume that $\partial E / \partial C$, the gradient of $E$ with respect to $C$, the cumulated penalty over the best path, is known. Since $C$ is simply the sum of the penalties of each arc in $G_{v i t}$, the gradients of $E$ with respect to these penalties are all equal to $\partial E / \partial C$. Since $G_{v i t}$ is a subset of $G_{r e c}$, the derivatives of $E$ with respect to the penalties in $G_{r e c}$ are equal to $\partial E / \partial C$ for those arcs that appear in $G_{v i t}$, and 0 for all others. The gradient of $E$ with respect to $w$, the parameter vector of the character scorer $R_{w}$ in $T_{r e c}$, is simply the sum over all arcs in $T_{r e c}$ of $\partial E / \partial C \partial R / \partial w$. We have done nothing more than apply the chain rule. The difference with traditional neural networks, and other systems in which gradient-based learning is commonly applied, is that the architecture (or dataflow graph) through which we propagate states and backpropagate gradients changes with the input data.

\subsection{Previous Work}

Over the last few years, many authors have proposed adhoc ways of building trainable systems by combining graphbased models with other learning methods such as neural networks, notably for speech recognition $[4,6]$, and handwriting recognition (see [2] for a review). However, there has been no proposal for a systematic approach to multilayer graph-based trainable systems. The idea of transforming graphs into other graphs has received considerable interest in computer science, through the concept of weighted finite-state transducers [11]. Transducers have been applied to speech recognition and language translation, and proposals have been made for handwriting recognition [5]. This line of work has been mainly focused on the algebraic aspects of combining transducers and graphs (called acceptors in this context), but very little effort has been devoted to building globally trainable systems out of transducers. Learning has been the focus of another line of work, called Input-Output HMM, which can model distributions over sequences conditioned on an input sequence [1]. The concept of building trainable systems by assembling modules and propagating gradients through them has been proposed for quite some time [3], and used extensively in practical systems [2]. However, it was not suggested that complex data structures such as graphs could be systematically used as state variables between modules.

\section{Reading Check Amounts with a Graph Transformer Network}

The idea of graph transformer networks was used to build a check amount reading system. The system must find candidate fields, select the field that is most likely to contain the amount, segment the fields into candidate characters, read and score the candidate characters, and finally find the best interpretation of the amount using contextual knowledge, namely a stochastic grammar for check amounts.

\subsection{A Graph Transformer Network}

We now describe the successive graph transformations that allow our network to read the check amount (cf. figure 3 ). Each Graph Transformer produces a graph whose paths encode and score the current hypothesis considered at this stage of the system. The input to the system a trivial graph whose only arc carries the image of the whole check.

THE FIELD LOCATION TRANSFORMER $T_{\text {field }}$ first performs classical image analysis (including connected components, ink density histograms, etc...) and heuristically extracts rectangular zones that may contain the check amount. $T_{\text {field }}$ produces an output graph, called the field graph (cf. figure ??) such that each candidate zone found is associated with one arc that links the start node to the end node. Each arc contains the image of the zone, and a "penalty term" computed from simple features extracted from the zone (absolute position, size, aspect ratio,...). The penalty term is close to zero if the features suggest that the field is a likely candidate, and is large if the field is deeemed less likely to be an amount. If the penalty function is differentiable, its parameter are globally tunable.

THE SEGMENTATION TRANSFORMER $T_{\text {seg }}$, similar to the one described in section 3. examines each zone contained in the field graph, and cuts each image into "pieces of ink" using heuristic image processing techniques. Each piece of ink may be a whole character or a piece of character. Each arc in the field graph is replaced by its corresponding segmentation graph that represents all possible groupings of pieces of ink. Each field segmentation graph is appended to an arc that only contains the penalty of the field in the field graph. Each arc carries the segment image, together with a penalty that provides a first evaluation 


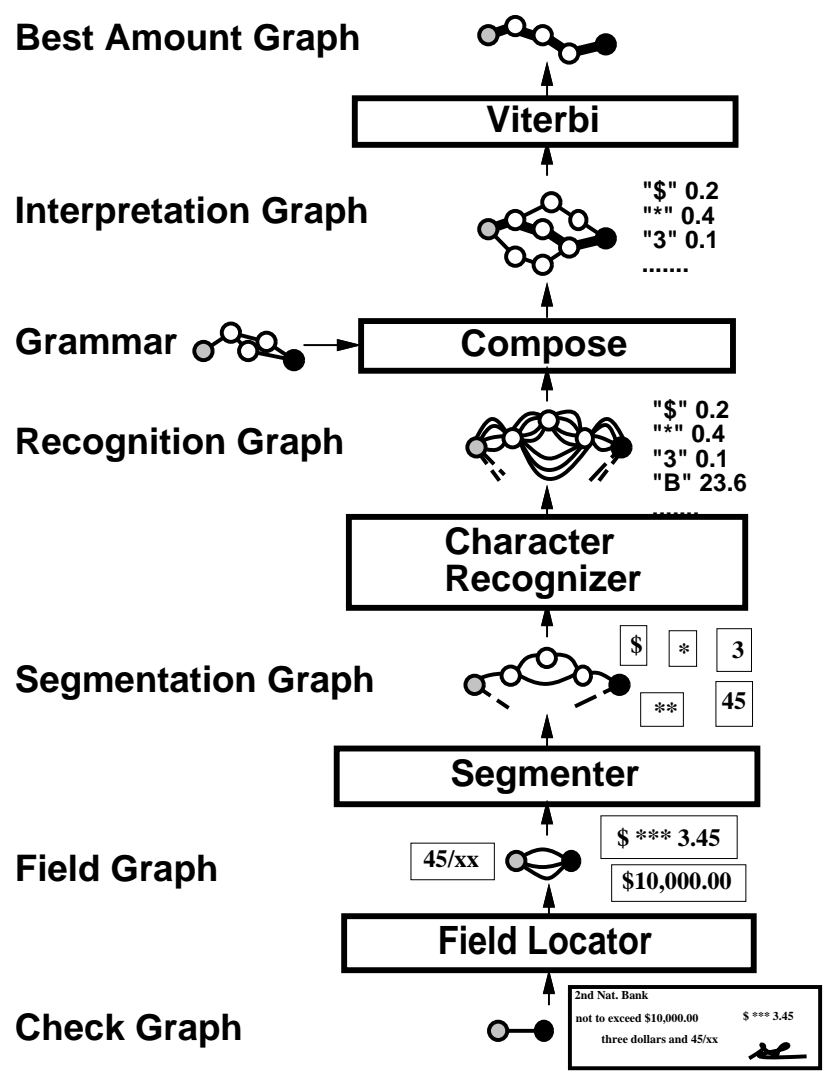

Fig ure 3. A complete check amount reader implemented as a single cascade of graph transformers.

of the likelihood that the segment actually is character. This penalty is obtained with a differentiable function that combines a few simple features such as the space between the pieces of ink, or the compliance of the segment image with a global baseline, using a few tunable parameters. The segmentation graph represents all the possible segmentation of all the field images. We can compute the penalty for one segmented field by adding the arc penalties along the corresponding path. As before using a differentiable function for computing the penalties will ensure that the parameters can be optimized globally.

THE RECOGNITION TRANSFORMER $T_{r e c}$ iterates over all segment arcs in the segmentation graph and runs a character recognizer on the corresponding segment image. In our case, the recognizer is a Convolutional Neural Network [8] whose weights constitute the largest and most important subset of tunable parameters. The recognizer classifies images into one of 15 classes (10 digits, asterisk, Dollar sign, dash, comma, period), plus "junk" classes for other symbols or badly-formed characters. Each arc in the input graph is replaced by 16 arcs in the output graph. Each of those 16 arcs contains the label of one the classes, and a penalty that is the sum of the penalty of the corresponding arc in the input graph and the penalty associated with classifying the image in the corresponding class, as computed by the recognizer. In other words, the recognition graph rep-

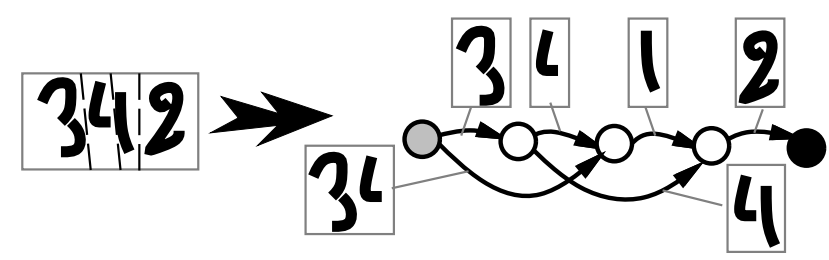

Figure 4. Each path in the segmentation graph represents one possible grouping of the "pieces of ink" into characters.

resents a weighted treillis of scored character classes. Each path in this graph represents a possible character string for the corresponding field. We can compute a penalty for this interpretation by adding the penalties along the path. This sequence of characters may or may not be a valid check amount.

THE GRAMMAR TRANSFORMER $T_{\text {gram }}$ selects the paths of the recognition graph that represent valid character sequences for check amounts. This transformer takes two graphs as input: the recognition graph, and the grammar graph. The grammar graph contains all possible sequences of symbols that constitute a well-formed amount. The output of the grammar transformer, called the interpretation graph, contains all the paths in the recognition graph that are compatible with the grammar. The operation that combines the two input graphs to produce the output is called a graph composition. This algorithm was formally studied in the context of finite-state transducers, and although its general form is quite complex, [11], it can be explained using a simple metaphor. To generate the interpretation graph we place a token on the start node of the recognition graph and a token on the start node of the grammar graph. We can simultaneously advance the two tokens along two arcs if the numerical information attached with the two arcs match according to a matching function. In our case, the matching function simply checks that the class labels on the two arcs are indentical. When the two tokens are moved, an arc in the output graph is created. A differentiable function is used to compute the data attached to the output arc from the data attached to the input arcs. In our case, the output arc receives the class label of the two arcs, and a penalty computed by simply summing the penalties of the two input arcs (the recognizer score, and the arc penalty in the grammar graph). The above procedure produces a tree, but simple techniques can be used to avoid generating multiple copies of certain subgraphs by detecting when a particular output state has already been seen. Each path in the interpretation graph represents one interpretation of one segmentation of one field on the check. The sum of the penalties along the path represents the "badness" of the corresponding interpretation and combines evidence from each modules along the process, as well as from the grammar.

THE VITERBI TRANSFORMER finally selects the path with the lowest accumulated penalty, corresponding to the best grammatically correct interpretations.

If a probabilistic score is desired, we can obtain one by computing the ratio between (a) the negative exponential of the total penalty of best path, and (b) the sum of the negative exponentials of the penalties of all the paths. Such path 
probabilities are clearly positive and sum to one. The denominator is easily computed using the forward algorithm widely used in Hidden Markov Model-based speech recognition systems [12]. We can directly compute its logarithm by proceeding forward in the graph and setting the penalty of each node to the logsum of the penalties of the incoming arcs added with the penalties of the upstream nodes. The penalty of the end node can be interpreted as the negative log-likelihood that the check contains a grammatically correct amount.

A similar procedure allows us to compute the negative log-likelihood of any amount we choose. For instance, if we compute ratio between (a) the sum of the negative exponential of the penalties of all paths representing the correct amount, and (b) the sum of negative exponential of the penalties of all paths, we will obtain the likelihood attributed by the system to the correct answer. This is particularly useful for global training as will be discussed below.

\section{Gradient-Based Learning}

Each stage of this check reading system contains tunable parameters. While some of these parameters could be manually adjusted, for example the parameters of the field locator and segmentor, the vast majority of them must be learned, particularly the weights of the neural-net recognizer.

Prior to globally optimize the system, the parameters of each module must be initialized with reasonable values. The parameters of the the field locator and the segmentor are easily initialized by hand. The parameters of the neural net character recognizer can be initialized by training on a database of pre-segmented and labeled characters. Then, the entire system can be trained globally from whole check images labeled with the correct amount. No explicit segmentation of the amounts is needed to train the system: it is trained at the check level.

The objective function $E$ minimized by our global training procedure is a discriminant criterion similar to the Maximum Mutual Information criterion used in speech recognition systems based on Hidden Markov Models [12]. This criterion is the difference between two penalties. The first penalty is the result of running the forward algorithm on the graph produced by composing the recognition graph with a grammar that only contains the correct answer. The second penalty is the result of running the forward algorithm on the graph produced by composing the recognition graph with the full grammar. Since the grammar includes the correct answer, the objective function is always positive. It is zero if the penalties of the wrong answers are infinitely larger than the penalty of the correct answer. For the training phases, we therefore pass the recognition graph through two transformers (a "constrained forward algorithm" and a "free forward algorithm") that output the corresponding negative log-likelihoods, the difference of which is $E$. The partial derivatives of $E$ with respect to the arc penalties in the interpretation graphs are simply computed by back-propagation. All the arc penalties along the paths representing the correct answer will have a positive gradient, while all other arcs will have negative gradients.

Once we have those, we back-propagate gradients through the grammar transformer and obtain partial derivatives with respect to the penalties on the recognition graph and on the grammar graphs. This can be done if we keep

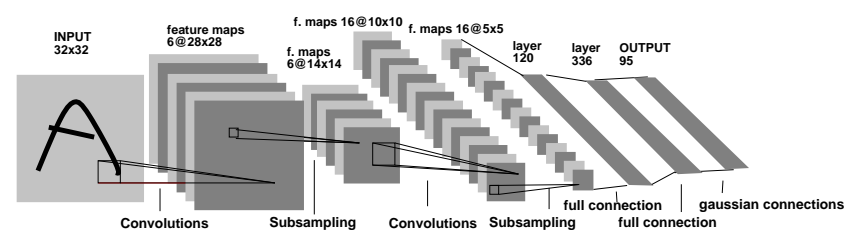

Figure 5. Architecture of LeNet 5. Each plane is a feature map, i.e. a set of units whose weights are constrained to be identical.

track of which arcs in the grammar and recognition were used to generate which arc on the interpretation graph. Since these penalties $s_{\text {int }}$ are simple combinations of the arc penalties of the grammar graph and of the arc penalties of the recognition graph, we can compute the derivatives $\partial E / \partial w_{\text {gram }}$ with respect to the tunable parameters of the grammar and the derivatives $\partial E / \partial s_{\text {rec }}$ with respect to the arc penalties of the recognition graph.

Since these penalties $s_{\text {rec }}$ are simple combination of the arc penalties in the segmentation graph and of the penalties returned by the character recognizer, we can first compute the derivatives $\partial E / \partial s_{\text {net }}$ with respect to the outputs of the character recognizer and therefore compute the derivatives $\partial E / \partial w_{\text {net }}$ of all tunable parameters of the recognizer. We can also compute the derivatives $\partial E / \partial s_{\text {seg }}$ with respect to all the penalties of the segmentation network.

Since these penalties $s_{\text {seg }}$ are simple combination of the arc penalties of the field graph and of the tunable parameters of the segmenter, we can compute the derivatives $\partial E / \partial w_{\text {seg }}$ with respect to the tunable parameters of the segmenter and the derivatives $\partial E / \partial s_{\text {field }}$ with respect to the arc penalties of the field graph.

Finally we can use these latter derivatives to compute the derivatives $\partial E / \partial w_{\text {field }}$ with respect to the parameters of the field locator.

In doing so, we have done nothing more than apply the chain rule, albeit in a somewhat complicated, and dynamic, architecture.

\section{Shape Recognition with Convolutional Neural Networks}

The recognizer used in the Check Reading System is a convolutional neural network coined LeNet5. Convolutional neural nets are specifically designed to recognize $2 \mathrm{D}$ shapes with a high degree of invariance with respect to translations, scaling, skewing, and other distortions. They can directly accept images with no preprocessing other than a simple size normalization and centering. They have had numerous applications in handwriting recognition [10, 2,9], and object location in images, particulaly faces [13]. The architecture of LeNet5 is shown in figure 5. In a convolutional net, each unit takes its input from a local "receptive field" on the layer below, forcing it to extract a local feature. Furthermore, units located at different places on the image are grouped in planes, called feature maps, within which units are constrained to share a single set of weights. This makes the operation performed by a feature map shift invariant, and equivalent to a convolution, followed by squashing functions. This weight-sharing tech- 
nique greatly reduces the number of free parameters. A single layer is formed of multiple feature maps, extracting different features types.

Complete networks are formed of multiple convolutional layers, extracting features of increasing complexity and abstraction. Sensitivity to shifts and distortions can be reduced by using lower-resolution feature maps in the higher layers. This is achieved by inserting subsampling layers between the convolution layers. It is important to stress that all the weights in such a network are trained by gradient descent. Computing the gradient can be done with a slightly modified version of the classical backpropagation procedure. The training process causes convolutional networks to automatically synthesize their own features. LeNet5 has 401,000 connections, but only about 90,000 free parameters because of the weight sharing.

\section{Results}

A version of the above system was implemented and tested on a representative mixture of business checks and personal checks. Existing heuristic algorithms have been reimplemented as graph transformers. The modular graph transformer architecture effectively separates the global system design and the application specific algorithms.

The neural network classifier was initally trained on 500,000 images of normalized character images from various origin spanning the entire printable ASCII set. This contained both handwritten and machine-printed characters. Additional images were generated by randomly distorting the original images using simple affine transformations of the images. The network was then further trained on characters that were hand-segmented from check images. It was then inserted in the check reading system and trained globally.

The performance of the system at the check level is $1 \%$ error, $50 \%$ correct, and $49 \%$ rejected. The test set was disjoint from the training set and composed of a "natural" mixture of business checks and personal checks. On business checks, which are generally machine-printed, the amount is relatively easy to read, but quite difficult to find due to the lack of standard for business check layout. On the other hand, the amount on personal checks is easy to find but much harder to read. The use of a grammar for check amounts seem particularly helpful for reading business checks.

Independent test by systems integrators have shown the superiority of this system over other commercial systems. The system was integrated in NCR's latest line of backoffice check reading machines. It was fielded in a bank on June 1996, and is reading millions of checks per month.

Because it is trainable, the system can be quickly adapted to new tasks, such as reading checks from other countries or reading other types of documents.

\section{Conclusion}

We have presented a new architecture for trainable systems that significantly extends the domain of application of gradient-based learning. We have shown that all of the steps of a document analysis system can be formulated as graph transformers through which gradients can be backpropagated. A Check Reading System, based on these ideas was built. It is presently deployed commercially and reads millions of personal checks and business checks per month with record accuracy.

Although this paper presents a small number of examples of Graph Transformer Modules, it is clear that the concept can be applied to many situations where the domain knowledge or the state information can be represented by graphs. This is the case in many scene analysis systems, and many applications of comuter vision. Future work will attempt to apply graph transformer networks to such problems, with the hope of allowing more reliance on automatic learning, and less on detailed engineering.

\section{References}

[1] Y. Bengio and P. Frasconi. An input/output HMM architecture. In G. Tesauro, D. Touretzky, and T. Leen, editors, Advances in Neural Information Processing Systems, volume 7, pages 427-434. MIT Press, Cambridge, MA, 1996.

[2] Y. Bengio, Y. LeCun, C. Nohl, and C. Burges. Lerec: A $\mathrm{nn} / \mathrm{hmm}$ hybrid for on-line handwriting recognition. Neural Computation, 7(5), 1995.

[3] L. Bottou and P. Gallinari. A framework for the cooperation of learning algorithms. In D. Touretzky and R. Lippmann, editors, Advances in Neural Information Processing Systems, volume 3, Denver, 1991. Morgan Kaufmann.

[4] H. Bourlard and C. Wellekens. Links between markov models and multilayer perceptrons. In D. Touretzky, editor, $A d$ vances in Neural Information Processing Systems, volume 1, pages 186-187, Denver, 1989. Morgan-Kaufmann.

[5] I. Guyon, M. Schenkel, and J. Denker. Overview and synthesis of on-line cursive handwriting recognition techniques. In P. S. P. Wang and B. H., editors, Handbook on Optical Character Recognition and Document Image Analysis. World Scientifi c, 1996.

[6] P. Haffner, M. Franzini, and A. Waibel. Integrating timealignment and neural networks for high performance continuous speech recognition. In Proc. of ICASSP 91. IEEE, 1991.

[7] Y. Le Cun and Y. Bengio. Convolutional networks for images, speech, and time-series. In M. A. Arbib, editor, The Handbook of Brain Theory and Neural Networks. MIT Press, 1995.

[8] Y. Le Cun, B. Boser, J. S. Denker, D. Henderson, R. E. Howard, W. Hubbard, and L. D. Jackel. Back-propagation applied to handwritten zipcode recognition. Neural Computation, 1(4), January 1990.

[9] Y. Le Cun, L. D. Jackel, L. Bottou, A. Brunot, C. Cortes, J. S. Denker, H. Drucker, I. Guyon, U. A. Muller, E. Sackinger, P. Simard, and V. Vapnik. Comparison of learning algorithms for handwritten digit recognition. In F. Fogelman and P. Gallinari, editors, International Conference on Artifi cial Neural Networks, pages 53-60, Paris, 1995. EC2 \& Cie.

[10] Y. Le Cun, O. Matan, B. Boser, J. S. Denker, D. Henderson, R. E. Howard, W. Hubbard, L. D. Jackel, and H. S. Baird. Handwritten zip code recognition with multilayer networks. In IAPR, editor, Proc. of the International Conference on Pattern Recognition, Atlantic City, 1990. IEEE. invited pa-

[11] F. Pereira, M. Riley, and R. Sproat. Weighted rational transductions and their application to human language processing. In ARPA Natural Language Processing workshop, 1994.

[12] L. R. Rabiner. A tutorial on hidden markov models and selected applications in speech recognition. Proceedings of the IEEE, 77(2):257-286, Februalry 1989.

[13] R. Vaillant, C. Monrocq, and Y. Le Cun. Original approach for the localisation of objects in images. IEE Proc on Vision, Image, and Signal Processing, 141(4), August 1994. 